\title{
PROMOCIÓN DE LAS EMOCIONES POSITIVAS EN LA ÚLTIMA ETAPA DE LA VIDA
}

\author{
Alba Vázquez-Blanco \\ Complejo Asistencial de Zamora \\ alba3108@gmail.com \\ Andrea Morán-Carrera \\ Complejo Asistencial de Zamora.
}

Recepción Artículo: 06 diciembre 2021

Admisión Evaluación: 06 diciembre 2021 Informe Evaluador 1: 07 diciembre 2021

Informe Evaluador 2: 07 diciembre 2021

Aprobación Publicación: 07 diciembre 2021

\section{RESUMEN}

El ser humano experimenta una inmensidad de emociones que son las que condicionan como nos encontramos en cada momento y estas se hacen más notables y vulnerables al final de la vida. Las emociones afectan en gran parte al progreso de nuestras vidas y determinan nuestras acciones ya que alteran la atención, la memoria y la motivación y además limitan nuestras conductas repercutiendo en nuestra salud, bienestar y, en definitiva, en nuestra calidad de vida. Es por ello que las emociones juegan un papel muy importante para la salud y por eso se tienen que cuidar aún más llegada la edad avanzada con el fin de potenciar una conducta saludable y así evitar un riesgo potencial para salud que repercutirá sustancialmente en el anciano. De modo que los sanitarios debemos trabajar en buscar terapias y talleres que transformen las emociones en estímulos, motivaciones y aprendizajes positivos. La educación emocional, persigue la prevención y reducción de las situaciones que inciden negativamente en la salud física y psíquica, para promover el equilibrio emocional y un verdadero estado de bienestar. Con esta comunicación se intenta proporcionar una sinopsis teórica y reflexiva acerca del desarrollo de las emociones como uno de los factores determinantes la salud y el bienestar de las personas de tercera edad.

Palabras clave: emociones; salud emocional; educación para la salud; ancianos; promoción de la salud; prevención; inteligencia emocional; bienestar

\section{ABSTRACT}

Promotion positive emotions in the last stage of life. The human being experiences an immensity of emotions that are the ones that condition how we find ourselves in each moment and these become more noticeable and vulnerable at the end of life. Emotions largely affect the progress of our lives and determine our actions since they alter attention, memory and motivation and also limit our behaviors, affecting our health, wellbeing and, ultimately, our quality of life. That is why emotions play a very important role for health and that is why 


\section{PROMOCIÓN DE LAS EMOCIONES POSITIVAS EN LA ÚLTIMA ETAPA DE LA VIDA}

they have to be taken care of even more in advanced age in order to promote healthy behavior and thus avoid a potential health risk that will have a substantial impact on the elderly. So we health workers must work to find therapies and workshops that transform emotions into positive stimuli, motivations and learning. Emotional education pursues the prevention and reduction of situations that negatively affect physical and mental health that allows promoting emotional balance and a true state of well-being. This communication is intended to provide a theoretical and reflective synopsis about the development of emotions as one of the determining factors of the health and well-being of the elderly.

Keywords: emotions; emotional health; health education; seniors; health promotion; prevention; emotional intelligence; well-being

\section{INTRODUCCIÓN}

Las especulaciones sobre la asociación entre la ciencia del cerebro y el bienestar se examinaron por primera vez con el análisis de Sigmund Freud a principios del siglo XX. Hoy en día, aunque la conexión entre los factores emocionales y el bienestar aún no está fundamentada, disponemos de un conjunto adecuado de pruebas lógicas que demuestran que esta relación existe, es bidireccional y tiene una premisa psicobiológica.

Hace más de 25 años surgió la idea de "la capacidad de comprender a las personas en su esencia" (IE), que comprende el reconocimiento del valor de los sentimientos y la posterior eliminación de la lógica y el cognitivismo.

Existe una relación positiva entre la IE y diferentes límites de bienestar o marcadores naturales, por ejemplo, la calidad del descanso, el nivel de glucosa en sangre, la alteración del pulso o el nivel de cortisol debido a la activación del eje hipotálamo-hipófisis-suprarrenal. Por lo tanto, la IE debe considerarse como un elemento que favorece el bienestar individual, ya que los sentimientos repercuten directamente en nuestro bienestar físico y psicológico.

La capacidad de comprender a cualquier persona en su esencia es una de las cualidades que, en equilibrio, conforman a un individuo importante y seguro, tanto a nivel individual como comparable a los individuos que le rodean. En este sentido, es la capacidad que necesitamos para hacernos cargo, comprender o manejar nuestros propios sentimientos y, en consecuencia, ser comprensivos con los sentimientos de los demás.

Mayer y Salovey, eminencias en esta fuerza, caracterizan la capacidad de comprender a las personas en su esencia como "la capacidad de ver, valorar y expresar los sentimientos de forma satisfactoria y adaptativa. La capacidad de obtener sentimientos, la utilización de activos entusiastas y la capacidad de dirigir sentimientos tanto en uno mismo como en otras personas".

Debido a la época en la que crecieron y a lo que se encontraron, muchos individuos de más de 60 años no han tenido la oportunidad de averiguar cómo percibir sus sentimientos o consideran que no es agradable comunicarlos o hablar de ellos. Esta es la situación, sobre todo, de los hombres más consolidados, que tienen un lugar con una edad que les obliga a ocultar su lado entusiasta. "Los hombres no lloran" era una premisa denostada para los chicos.

Por eso, Ilegar a la edad avanzada sinceramente sólido, saber ver el valor en el presente, aclimatarse a cada segundo, ajustarse al clima y, obviamente, fomentar una metodología suelta y esperanzadora es inseparable de una maduración dinámica y eficaz. La sociedad actual refleja una perspectiva negativa de los mayores, en la que la maduración se relaciona con la decadencia real, la disminución de las capacidades intelectuales, lo que propicia circunstancias de reclusión social, desamparo y, en consecuencia, irregularidad pasional que acaban influyendo en la fuerza orgánica de los mayores, con más implicaciones negativas que buenas. La gran capacidad de comprender a las personas a nivel profundo en la vejez se convierte en una satisfacción personal superior y nos orienta hacia una maduración sólida.

El nivel que debemos tener como expertos en atención médica y como figuras parentales en todos los círculos de la vida de los ancianos es cambiar esta visión de las desgracias por ganancias, alabar las victorias y no las decepciones, destacar la experiencia valiosa y no la miseria, para decirlo claramente, sacar a la luz los problemas, prevenir y enseñar en la mejora de este límite apasionante. A medida que los individuos avanzan en el 
camino de la vida, el bienestar se sitúa como una columna esencial para seguir siendo dinámicos y mantener la independencia, por lo que debemos alabar este pensamiento desde el principio para convertirlo en una propensión para siempre.

Las habilidades que conforman la capacidad de apreciar a cualquier persona en un nivel profundo incluyen ver y percibir los sentimientos, tanto los propios como los de los demás, supervisarlos y utilizarlos de forma admirable. En la actualidad, la capacidad de apreciar a las personas a un nivel profundo se compone de diferentes habilidades que se han obtenido o preparado a lo largo de la vida. Entre ellas, merece la pena centrarse en:

- Impresión de los sentimientos, es la capacidad de ver los sentimientos propios y los de los demás, hay muchos individuos que no tienen ni la más remota idea de cómo separar bien entre estar desanimado, sentir pavor, tensión, estar iracundo, etc. Además no darse cuenta de cómo separarlos no nos permite comunicar nuestro mundo interior de forma efectiva, lo que influye en la naturaleza de nuestras conexiones.

- Compasión, aludimos a la capacidad de ponernos en el lugar del otro individuo. Ver cómo se sienten, respecto a sus sentimientos y las explicaciones de su conducta.

- La asistencia es la capacidad de utilizar los sentimientos en nuestra vida para simplemente decidir, ocuparnos de los asuntos, mirar a través de nuestra memoria, etc.

- La disposición entusiasta es la capacidad de obtener sentimientos y ganar destreza con sus implicaciones. Nos ayuda a comprender cómo descifrar nuestros propios sentimientos y los de los demás, y a controlar la relación y el progreso empezando por un sentimiento y luego por el siguiente.

- La orientación entusiasta depende de la capacidad de manejar los sentimientos, tanto los buenos como los negativos, para que no afecten contrariamente a nuestra prosperidad. No enfadarse, no disgustarse, no inquietarse por cuestiones pasajeras o que tienen respuesta es una empresa fundamental para nuestro autoconocimiento.

Para decirlo claramente, a través de la preparación de estas habilidades podemos averiguar cómo dominar nuestros sentimientos y no dejar que gobiernen nuestra rutina habitual. Este es el objetivo razonable de los expertos en atención médica para fortalecer sinceramente a los mayores, ya que la fase de la vida conocida como la tercera edad requiere una consideración y un cuidado explícitos. En este sentido, los tratamientos para la tercera edad buscan llevar una satisfacción personal ideal a los ancianos y aplicarlos de inmediato y desarrollarlos con constancia asegura aparatos para hacer frente a cada una de las circunstancias que surgen en este periodo.

El objetivo principa/ de este trabajo de investigación es destacar la importancia de la capacidad de comprender a cualquier persona en su núcleo en la maduración dinámica, para concentrarse en sus habilidades y en los círculos a los que están conectados y cómo canalizar los sentimientos negativos.

El objetivo secundario es descubrir cómo se puede asociar a los expertos en bienestar a la hora de impartir la escuela del bienestar, para averiguar hasta qué punto se consideran los sentimientos como una pieza vital del bienestar y la prosperidad de los mayores.

\section{MÉTODO}

Se ha llevado a cabo una revisión bibliográfica de artículos científicos concretamente publicados por expertos en la materia, como por ejemplo psicólogos sanitarios con especialidad en neuropsicología y desarrollo cognitivo en personas mayores, psiquiatras que tratan a personas geriátricas, pedagogos, trabajadores sociales de centros geriátricos y potenciadores de la educación emocional.

Los artículos que se han usado para realizar este estudio tienen una antigüedad inferior a siete años y están publicados en lengua española.

\section{RESULTADOS}

La fase de la edad avanzada se inicia con el periodo de jubilación, de deserción de la vida laboral dinámica. Esto sugiere que, en general, puede haber una inclinación a caer en un modo de vida inactivo y en la abulia, ya que generalmente no estamos preparados para darnos cuenta de cómo utilizar nuestra energía sobrante de forma 


\section{PROMOCIÓN DE LAS EMOCIONES POSITIVAS EN LA ÚLTIMA ETAPA DE LA VIDA}

proactiva. Saben que algo no funciona de forma adecuada y que soportan escenas de confusión o que su propia independencia disminuye.

Durante la edad avanzada debemos reforzar nuestro bienestar y tener la opción de enfrentarnos a los puntos difíciles, ya que, durante esta fase de la vida, nos volvemos más delicados, más propensos a las condiciones médicas mentales y reales y a los límites. Ejemplos de estas limitaciones pueden ser la presencia de enfermedades, problemas más destacados de recuperación, ausencia de tareas proactivas, segregación social, ausencia de inspiración...

Hay que recordar que las progresiones en esta etapa son inesperadas y conciernen a numerosas regiones: de otra circunstancia monetaria a una reorganización en cuanto a la vida respectivamente. Para referirnos a un modelo, la jubilación de uno de los individuos Ileva consigo nuevos horarios y menos sueldo. Otro modelo es el momento en el que los mayores acaban en la situación de criar a sus nietos, lo que les quita energía extra, o cuando sus problemas médicos les Ilevan a cambiar de casa ya que actualmente no pueden cuidar de sí mismos.

El modelo integral de bienestar requiere recordar los sentimientos de anticipación y las metodologías de avance. Llegar a la tercera edad con una adaptabilidad apasionada garantiza la seguridad, la confianza, la prosperidad y, obviamente, la mejor idea de la felicidad. Las investigaciones en este campo relacionan esta fortaleza con otras cualidades adicionalmente enormes como la flexibilidad o el sentido imperativo, no dejemos de recordar que en las sociedades, por ejemplo, orientales donde gana la tranquilidad, el positivismo entusiasta y se desarrolla la preparación mental y el sentido crucial son más longevas y alegres. (García y Miralles, 2016).

Según la revisión dirigida en la Universidad de Yeshiva, que probó estas ideas reafirmando la investigación pasada, fue a la seguridad de que los individuos aparentemente perpetuos comparten dos perspectivas vitales de la vida normal: positivismo y alta expresividad entusiasta. Las cualidades como características de carácter están disponibles en nuestra satisfacción personal de manera tan excepcional que como expertos en servicios médicos necesitamos actualizar estas cualidades, instruir durante la duración del ciclo de la existencia de manera apasionada para aceptarlo como una propensión de vida una vez entrado en la tercera edad para lograr individuos más alegres más experimentados en el ojo público y con su propia circunstancia actual. Así, el individuo a lo largo de su vida crea activos y cuando la persona llega a esta reunión de edad, la persona tiene una comprensión superior de sus cualidades en general y del sistema de maduración específicamente. En síntesis, lo que podría conocerse como la teoría de la integración cognitivo- emocional recomendaría que los ancianos, considerando factores reales que no pueden ser cuestionados por los mayores, optan por favorecer las emociones positivas como clave de equilibrio, felicidad y deseabilidad social.

Posteriormente, para aplicar y llegar al final de la hipótesis de la coordinación intelectual pasional, es excepcionalmente importante tratar la capacidad de comprensión de los individuos a nivel profundo en la edad avanzada, ya que es una fase rebosante de dificultades inevitables. Ejemplos de ello son la disminución real, cierta desintegración de las capacidades intelectuales, la desvinculación social, la propensión a la enfermedad... que afectan negativamente a la prosperidad de los ancianos. A esto hay que añadir las desgracias entusiastas, Ios problemas para reintegrarse en la sociedad y la disminución de los activos financieros.

En este contexto, es importante saber cómo manejar las progresiones que acompañan a la edad. Este manejo apasionado debe ir unido a una ayuda amistosa y a una tonelada de inspiración interior para ver el valor del presente, para lo cual es fundamental el dominio de la capacidad de apreciar a las personas en un nivel profundo.

No obstante, un gran número de nuestras personas mayores no comunican los sentimientos creados por ciertas circunstancias que necesitan para mirar en esta etapa imperativa. Tampoco tienen la menor idea de cómo controlar y elegir la naturaleza de sus consideraciones, permitiendo que las contemplaciones tontas o negativas inunden sus vidas.

El punto de vista psicoterapéutico o disposición de los problemas internos coincide con la línea instructiva más centrada en la anticipación. Ambas se dan, obviamente, en función de la situación concreta, pero parece que 
la parte instructiva necesita más peso, cuando una ampliación de este tipo de intercesión daría un resultado preventivo que, sin duda, disminuiría la necesidad de la última opción.

Según Bisquerra (2000), la formación pasional es un ciclo instructivo, persistente y extremadamente duradero, que espera potenciar el perfeccionamiento entusiasta como complemento vital del giro intelectual, estableciendo ambos los componentes fundamentales del avance del carácter esencial. Para ello, se propone el avance de la información y las habilidades sobre los sentimientos para capacitar a la persona para afrontar con mayor facilidad las dificultades que surgen en la existencia diaria. Esto apunta a ampliar la prosperidad individual y social.

Enseñar a apreciar a las personas a un nivel más profundo implica hacerles conscientes de cómo los pensamientos afectan a sus sentimientos y repercuten directamente en su bienestar. Sin embargo, también implica darles recursos para que sepan cómo adaptarse a los cambios y no pierdan la ilusión de ir minando su autoconciencia.

Por otra parte, es imprescindible destacar un elemento más de increíble pertinencia en lo que respecta a la comprensión y atención del universo entusiasta de los mayores. Aludimos a la actual inclinación social a ver la madurez como una fase de simple debilidad o inadecuación. Sin duda, esta peculiaridad sociocultural, habitual en las culturas actuales, también puede afectar realmente a los adultos más consolidados. Sin duda, la discriminación por edad y la minimización social de los ancianos afectan negativamente a su mente y a su confianza, excepto si sus sentimientos son sólidos y se dan cuenta de cómo equilibrar esta dificultad imperante con una capacidad inatacable de comprender a la gente en un nivel profundo.

Y es que una gran administración del mundo emocional disminuye la presión, las contemplaciones negativas, la pena y la decepción. Además, permite a los adultos más consolidados afrontar con mayor probabilidad las dificultades diarias de esta fase de la vida de alto nivel. Una ventaja más de enseñar la capacidad de apreciar a las personas a un nivel más profundo en la tercera edad es que trabaja con la variación a las nuevas circunstancias, la socialización y la adaptación a los problemas.

Además, al instruir en la capacidad de apreciar la inteligencia emocional, descubrimos cómo reforzar el bienestar emocional y prevenir la mejora de problemas mentales como la tristeza o el nerviosismo. Asimismo, aumenta la confianza y trabaja en las habilidades amistosas y la naturaleza de las conexiones relacionales. Para ello, se pueden planificar, ejecutar y evaluar los programas de educación para el bienestar que incorporan el entrenamiento pasional, así como evaluar los proyectos de instrucción entusiasta mediante la coordinación de medidas psicométricas y biométricas (límites) del bienestar físico y emocional.

En consecuencia, la ejecución de los tratamientos a partir de los 65 años se considera una actividad fundamental para mantener la prosperidad biopsicosocial individual. El abanico de ofertas terapéuticas es realmente amplio y cada individuo las consolidará según sus propias necesidades. Lo importante es destinar tiempo y obligación individual para ensayarlas.

Los tratamientos más sugeridos para este encuentro son los mentales, los relacionados con la palabra, los sociales y los que se identifican con el trabajo activo. Cada uno de ellos repercute en el otro, y cada uno de ellos trabaja para aumentar o mantener la satisfacción personal del adulto más establecido. Además, una parte de su labor consiste en ayudarles a ensayar una vida sana y activo, que enriquezca su existencia.

Las terapias psicológicas, la psicoterapia en la tercera edad se ocupa de las necesidades particulares de esta etapa. En este tipo de tratamiento se trabajan los problemas de confianza, el tipo de vida que lleva el individuo y su cuidado. Además, se tiene en cuenta la ayuda social del individuo y qué tipo de conexiones amistosas existen en su vida.

Los propósitos detrás de la discusión en su mayor parte introducidos por personas más establecidas se identifican con cuestiones de abatimiento, pérdida, tristeza o cambios en sus vidas que requieren reordenamiento. Sea como fuere, también hay retos relacionados con su cómplice, su familia o su clima. Estas condiciones abren a los mayores a problemas de nerviosismo o estrés. 


\section{PROMOCIÓN DE LAS EMOCIONES POSITIVAS EN LA ÚLTIMA ETAPA DE LA VIDA}

Por otra parte, el comienzo de determinadas enfermedades, especialmente neurológicas, afecta inequívocamente al bienestar psicológico de los ancianos. Saben que algo no va bien, que experimentan los efectos nocivos de las escenas de confusión o que su propia independencia se está reduciendo. Este tipo de ciclos pueden ser muy horrendos y es fundamental que un experto en ciencias del cerebro les ayude a supervisarlos.

Los tratamientos relacionados con la palabra son extremadamente útiles para practicar las capacidades motoras finas de los individuos. Esto refuerza su capacidad para realizar ejercicios esenciales de la vida diaria de manera más eficaz y afecta decididamente a mantener o trabajar en su propia independencia.

La verdad es que los problemas de capacidades finas coordinadas se deterioran durante la edad avanzada, lo que es claro en dos tipos de circunstancias, lo más importante. En primer lugar, en aquellos individuos con problemas intelectuales de introducción; para esta situación, estas actividades ayudan a frenar su deterioro. En segundo lugar, en adultos más consolidados con enfermedades articulares, como el dolor de articulaciones o la artrosis, entre otras.

Los tratamientos relacionados con la palabra tienen un lugar inconfundible en los proyectos de ayuda de los centros de día y las residencias de ancianos. Su valor para lograr la prosperidad de los ancianos está profundamente demostrado y diferenciado. Su formación depende de ejercicios imaginativos o de animación intelectual que activan la aptitud manual. Por lo tanto, hacer, tocar instrumentos, hacer crucigramas o sudokus, etc., incluyen un lugar dentro de los tratamientos relacionados con la palabra.

En consecuencia, es fundamental que su aplicación dependa de las preferencias e intereses secundarios del adulto. Suponiendo que el adulto se vea obligado a realizar ejercicios que no son como ellos prefieren, su impacto podría ser contraproducente. En consecuencia, o bien el adulto no participará, o bien podría producir un sentimiento de decepción en él/ella.

Sea como fuere, una parte de los ejercicios relacionados con la palabra debe tener como objetivo enseñar nuevos encuentros 0 aprendizajes. De este modo, se satisfará el interés y la necesidad de variedad de los individuos. Pero, al mismo tiempo, sus capacidades cerebrales se animan al presentarles nuevas situaciones y nueva información.

Así pues, a pesar de la conveniencia de estos tratamientos como ejercicio real, su increíble recurso reside en la fastuosidad y plenitud que aportan.

Las recuperaciones basadas en el ejercicio piensan en el trabajo real como una forma de garantizar un gran bienestar físico y emocional. Su actividad se centra en el ejercicio real del trabajo motor grueso. Incorporan actividades de oposición moderada para reforzar los músculos, actividades articulares para desarrollar aún más el flujo en los apéndices y trabajos de extensión.

Por otra parte, las recuperaciones activas pueden basarse en ejercicios de alto impacto, largos paseos o actividades que consolidan la respiración y el desarrollo. La motivación principal de estos tratamientos es mantener el dinamismo del adulto, mejorar sus capacidades corporales y ampliar su prosperidad psicológica.

Además, este tipo de terapias son extremadamente convincentes para prevenir formas de vida estacionarias y ayudar a dirigir el resto de los mayores.

Los terapias alternativas han reforzado, sin duda, la cantidad de tratamientos para los mayores. Independientemente de que sean para individuos sanos con independencia individual, como si están destinados a individuos subordinados.

Las terapias con más difusión y aceptación para los mayores son las siguientes:

- Fisioterapia. Imposible de prevenir para ayudar a la recuperación del bienestar en caso de lesiones extremas, mediaciones cuidadosas o debilitamiento de enfermedades. Gracias a estos tratamientos utilitarios de recuperación, el individuo puede recuperar su portabilidad, esencialmente en general. Además, son excelentes para mantener la mejor independencia concebible y posponer el desmoronamiento real.

- Tratamiento musical. La música es excepcionalmente increíble para astillar las perspectivas, por ejemplo, la prosperidad entusiasta, la memoria y la socialización. Su utilización en los ancianos tiene resultados excep- 
cionalmente seguros. Dependiendo de sus destinos, puede calmar la mente o ser un motor que vigorice la acción física y psico-entusiasta de los individuos.

- Tratamientos táctiles. Su capacidad es la de vigorizar las facultades para prevenir su debilitamiento o calmar los estados de inquietud. Generalmente se utiliza para reeducar los ajustes sociales o para apoyar la memoria si se produce una demencia. Entre estos tratamientos, destaca la curación aromática.

- Risoterapia: conocido como hueso de la risa productivo, un método para tratar el estrés y la confianza a pesar de la dificultad. Numerosos clínicos han considerado que la inclinación cómica es una fijación fundamental en el bienestar emocional, ya que disminuye la presión, levanta la mentalidad y se llena como una válvula de seguridad para aliviar la tensión. A pesar de que el humor no cambia las realidades actuales, sirve para recontextualizar el punto de vista e impulsa la transformación positiva al cambio limitado. Spencer (1860), fue uno de los principales investigadores genuinos en insinuar los impactos ventajosos de la risa como componente fundamental para restablecer el solaz actual, la concordancia natural y el oído interno.

Las terapias socializadoras, durante la tercera edad debemos apostar por actividades que potencien las relaciones personales. Ya que, por diversas afecciones, muchos individuos de edad avanzada suelen desprenderse de los demás y llevar su vida a un abatimiento problemático que será el inicio de futuros desánimos y ausencia de inspiración para seguir con la vida en toda su calidad.

Aparte de las terapias psicológicas, ocupacionales o físicas que pueda llevar a cabo el mayor en grupo, hay otra serie de actividades. Por ejemplo, las direcciones, los viajes, los conjuntos, la inversión en tertulias, etc. Éstos potencian la conexión social y aportan una parte decente de relajación dinámica a las existencias de los ancianos. De ahí que, con el tiempo, acaben satisfaciendo una capacidad reparadora de prosperidad pasional.

En este sentido, el logro útil de estos ejercicios se muestra en una seguridad e inspiración más prominentes para hacer que cada momento cuente.

Las manualidades tienen un valor adicional, a la luz del hecho de que los mayores obtienen una infusión de confianza y valor individual ya que están explicando un trabajo que impartirán a otras personas e incluso proporcionarán a sus familiares y simultáneamente están apoyando los vínculos individuales, esta actividad tan evidentemente básica, les da una gran cantidad de autoafirmación y los asocia con su motivación a lo largo de la vida cotidiana. Por ejemplo, las marcas de Navidad se hacen decididas a regalarlas a sus hijos, nietos...

Los talleres de manualidades son multifuncionales y flexibles, ya que permiten el desarrollo de las manos en busca de un resultado innovador y aportan diversas ventajas. En este sentido, las creaciones para mayores son un activo fabuloso para avanzar en la maduración efectiva, ya que:

- Permiten mantener el cerebro dinámico y entrenar las capacidades intelectuales, ya que requieren consideración, concentración, precisión, etc.

- Accionan capacidades táctiles como la visión o el contacto. La necesidad de consolidar matices o materiales sirve para vigorizar las facultades.

- Aportan una tonelada de desenvoltura, por lo que son ideales para combatir estados de inquietud o sentimientos negativos. Asimismo, esperan que sepan sobre lo que están haciendo, lo que puede ser una instantánea de reflexión, con todas las ventajas que ello conlleva.

-Son un rico manantial de ejercicio real. Al realizar trabajos artesanales, Ios mayores ejercitan sus habilidades y capacidades reales, especialmente las de los apéndices superiores. Desde este punto de vista, están firmemente relacionados con los ejercicios de psicomotricidad fina.

- Apoyan la capacidad de innovación de los mayores. Un espacio fundamental de extrema importancia para la prosperidad individual. Cuanto más destacada sea la capacidad de innovación, más prominente será la capacidad de crear pensamientos rompedores, de buscar respuestas para circunstancias confusas y de tener un comportamiento adaptable, abierto a los cambios. 


\section{PROMOCIÓN DE LAS EMOCIONES POSITIVAS EN LA ÚLTIMA ETAPA DE LA VIDA}

- Avanzan en la eficiencia individual, algo que necesitan los individuos más consolidados para trabajar su confianza, su autoidea y su inspiración. Darse cuenta de que son útiles significa darse cuenta de que son valiosos y percibir que realmente tienen capacidades, dones y habilidades que compartir.

- Así se crean conexiones y combinaciones amistosas. Ya que la mayor parte de estos ejercicios se realizan en reuniones o tienen un motivo social, lo que hace que cada individuo contribuya con su trabajo para una ventaja típica.

De este modo, se potencia su propia utilidad e inspiración para participar o trabajar juntos en ejercicios de reunión o sociales. Es más, es una gran parte de la forma de pensar que hay detrás del envejecimiento activo y saludable. En concreto, que los mayores trabajen en su propia prosperidad, ya que encuentran razones que interactúan con su motivación a lo largo de la vida cotidiana.

No obstante, es esencial subrayar la importancia de realizar programas de mediación psicosocial con los propios residentes. El éxito de estos proyectos depende de la inclusión de los ancianos en ellos. En consecuencia, antes de llevar a cabo cualquier movimiento o estudio, es imprescindible conocer las preferencias, los supuestos y el carácter de los miembros. No basta con tener en cuenta sus necesidades fundamentales. Por ello, la consideración de su carácter y personalidad se considera un requisito incuestionable para la realización de cualquier movimiento útil. De ello depende que los ejercicios puedan producir un efecto positivo en su autoconciencia, que es lo que realmente buscamos.

En definitiva, y como objetivo, podemos atestiguar que la inteligencia emocional en la vejez resulta clave para su bienestar. Gracias a ella, es posible llevar a cabo una tercera edad más vital, saludable, más integrada en la sociedad y, obviamente, más plena y satisfactoria. Si nuestra esperanza de vida aumenta cada vez más, la inteligencia emocional se convierte, en la etapa de madurez, en un instrumento para participar en una mayor satisfacción personal y mejor calidad de vida.

Y es bajo la psicología, y en particular, bajo la psicogerontología recae el diseño de intervenciones destinadas al manejo eficaz de las emociones y una adecuada educación emocional, ofreciendo así una de las medidas más importantes para aumentar la probabilidad de envejecer de forma exitosa.

\section{DISCUSIÓN}

El equipo profesional de enfermería en las residencias de tercera edad, centros sociosanitarios o en cualquier consulta o contacto con la persona mayor, sobre todo en zonas rurales, debemos ejercitar la gestión emocional como herramienta de empoderamiento, motivación interna y actitud positiva que repercutirá en su biosfera global.

Para ello debemos de realizar actividades psicosociales con las que se mejore la inteligencia emocional en los ancianos. Actividades que se deben de realizar en un ambiente inclusivo, que genere bienestar e ilusión y fomente una vida social y personal rica en emociones sanas.

En conclusión, promover una madurez emocionalmente inteligente y positiva en las personas mayores se traduce en un envejecimiento activo y exitoso.

Como se puede apreciar, gracias a los talleres de manualidades y psicoterapias para personas mayores, se incrementa su salud integral y su motivación.

\section{REFERENCIAS BIBLIOGRÁFICAS}

López-Pérez, B., Fernández-Pinto, I., Márquez-González, M. (2008). Educación emocional en adultos y personas mayores. Electronic Journal of Research in Educational Psychology. 6(2).

Ortega Navas, MC (2010). La educación emocional y sus implicaciones en la salud. Revista Española de Orientación y Psicopedagogía, Vol.21, n²2, págs. 462-470.

Bisquerra Alzina, R. (2010). Educación emocional y competencias básicas para la vida. Revista de educación educativa, Vol. 21, no¹, págs. 7-43. 
Organización Mundial de la Salud. (4 de Noviembre de 2021). Envejecimiento y ciclo de vida. https://www.who.int/ageing/features/faq-ageism/es/

Perez- Fuentes, M.C., Molero, M.M., Gázquez, J.J., Soler F.J. (2014). Estimulación de la Inteligencia Emocional en mayores: El programa PECI-PM. European Journal of investigation in health, psychology and education. 4 (3), 329-339.

Orejudo Hernández S., Royo Más F., Soler Nagés JL. y Aparício Moreno L. (2014). Inteligencia emocional y bienestar. Reflexiones, experiencias profesionales e investigaciones. Universidad de Zaragoza.

Delhom Peris, I. (2019) Inteligencia emocional durante el envejecimiento. [Tesis doctoral, Universidad de Valencia].

https://roderic.uv.es/bitstream/handle/10550/70912/Tesis\%20lraida\%20Delhom\%20(1).pdf?sequence=1 
